\title{
El Saber de la Teoría de la Educación. Su ubicación conceptual
}

\author{
ANTONIO J. COLOM CAÑELlAS \\ Universidad de las Islas Baleares
}

\begin{abstract}
SUMMARY.-In this article is established the Theory of Education as a pragmatic theory or theory for practice. Also is distinguished the Theory of Education from Pedagogy and from others theoreticals studys of education. Its areas of application are determinated (formal, no formal and informal) and it is concluded with its own technological applicated caracteritation, common to any under which is pretended to analyses the theoretical question of education.
\end{abstract}

\section{ANTEAYER}

Debemos remontarnos a los últimos años del siglo XVIII así como a los primeros del XIX, para encontrar el referente «Pedagogía» aplicado al saber (experiencial o racional, especulativo o normativo) acerca de la educación. Por lo general, y en estos primeros años, el concepto de «Pedagogía» no se aplica aún a monografías o libros más o menos sistemáticos sino que se conforma como el último de los capítulos de las obras sobre Historia de la Educación, a modo de apéndice que trataba de la situación «actual» o última de la educación.

Tradicionalmente se cree que fue E. Kant quien utilizó por primera vez «Pedagogía» para referirse al conocimiento o reflexión de la educación, en sus primeros cursos dictados en 1.776. A partir de aquí Trapp (que ocupó la primera cátedra universitaria de Pedagogía en Halle), junto con los filantrópicos (seguidores de Bassedow), Nyemeyer, y por supuesto Herbart, irán configurando un cuerpo de «conocimiento pedagógico» (acerca de la educación) de cada vez más coherente, ordenado lógicamente y por tanto ya sistematizado.

En su origen, Pedagogía lo será todo; todo, claro está, que se refiera o tenga que ver con la educación. Pedagogía será pues el saber teórico y práctico, concreto o relacionado (la escuela, la familia...) con la educación. Más tarde, a principios del siglo XX, se producirá la primera gran desmembración que sufrirá este «todo» que por cierto será más de carácter metodológico, o de enfoque, que no de «espacio o topología del saber». Así, de la aplicación del método experimental surgirá la Pedagogía Experimental (Lay, Neumann...) o intento de dar cuenta del saber educativo en base al método científico, y de la tradición de entender el universo educativo desde la reflexión y la racionalización espe- 
culativa, se seguirá manteniendo la línea herbartiana, acaso ahora, evidenciada a través de diversas adjetivizaciones, ya que la Pedagogía que Herbart calificó de General (el objetivo del autor era presentar las ideas generales acerca de la educación), se reconocerá ahora por Racional (Paulsen), para así informarnos del aparato metodológico utilizado en su elaboración, por Fundamental (Cohn, Zaragueta...) o Esencial para indicarnos lo más importante o básico de la educación humana; por Sistemática (Gottler, Henz...) para informar de la coherencia o lógica interna del saber educativo, o por crítica si se encuentra sometida a revisión, a fin de lograr un cuerpo de conocimiento coherente y certero.

La Pedagogía se clasificó, entonces, de general, fundamental, esencial, sistemática o crítica, aunque todas estas denominaciones pretendieron, por una parte, presentar el conocimiento acerca de la educación para así fundamentar un modelo de educación válido para cualquier hombre -lo que debe estar presente en cualquier desarrollo educativo, o si se quiere, lo que conviene al educando-y por la otra, diferenciarse por su metodología de elaboración de la Pedagogía Experimental.

\section{AYER}

A partir de los años treinta de nuestro siglo, tiene lugar la segunda y más importante desmembración del cuerpo de conocimientos propio de la Pedagogía, desmembración que ahora afectará no tanto a la metodología cuanto a los contenidos, al saber pedagógico en suma. Las diferentes ciencias humanas, en su fulgurante desarrollo, coincidirán en considerar la educación como un espacio propio de sus respectivos estudios, por lo que la Psicología, la Sociología, la Filosofía, la Antropología, y más tarde, la Economía... etc. afectarán con sus aportaciones al conocimiento de la educación. Lo mismo sucederá con otras ciencias o desarrollo disciplinarios (Medicina, Psiquiatria, Biología, Ecología, Derecho...) que también, aunque parcialmente, adecuarán enfoques propios al saber educativo. Tras la Segunda Guerra Mundial la atomización de la ya antigua Pedagogía será un hecho, que se acrecentará hasta nuestros dias. Hoy, efectivamente, el saber pedagógico se encuentra en manos de las denominadas Ciencias de la Educación, conjunto múltiple de disciplinas propias (Didáctica, Organización Escolar, Historia de la Educación) y foráneas, que tienen en común estudiar aspectos de la educación, y que antes eran contemplados unitariamente por la Pedagogía. Sin más y a modo de resumen, podemos decir que la denominación «Ciencias de la Educación» se centra analítica y pluralmente en el mismo objeto de conocimiento -la educación- que antes era abordado sintética y unitariamente por la Pedagogía. No obstante hay que decir también que este cambio conlleva asimismo transformaciones metodológicas pues de la reflexión conceptual propia de la Pedagogía, se pasará ahora a un tratamiento plurimetodológico de las Ciencias de la Educación, más próximo a los tratamientos científicos de las demás ciencias humanas.

Entre las diversas ciencias de la educación nos encontramos con la denominada Teoría de la Educación, de origen anglófilo, y que se ha ido imponiendo entre nosotros por la gran incidencia lingüística que el inglés posee en estos momentos en todos los planos no sólo de la ciencia sino de cualquier otra esfera de la actividad humana (diplomacia, negocios, cultura... etc.); ahora bien, en inglés como se sabe, «education» hace referencia y se plantea como «practice», por lo que al querer abordar el estudio teórico de tal 
práctica y no poseer originalmente en su vocabulario el término de origen grecogermánico que nosotros reconocemos por Pedagogía, y que tradicionalmente se aplicaba al conocimiento teórico (léase racional, fundamental, sistemático, general... etc. de la educación), no queda más remedio que para significar tal tipo de estudio se tenga que echar mano del vocablo "Theory".

Vistas así las cosas, parece que Theory of Education es una de las múltiples ciencias de la educación que tiene como objeto el estudio teórico de la educación, por lo que de hecho se igualaría a la concepción de la clásica Pedagogía, que por cierto ya fue definida por Kant como "teoría de la educación". O sea, que una disciplina surgida de la vocación analítica de las ciencias de la educación y como una más de las mismas, aportaría una visión de síntesis, unitaria y general de la educación desde una perspectiva teórica (como la antigua Pedagogía), lo que por otra parte no dejaría de ser una curiosa paradoja.

Sin embargo, antes de llegar a conclusiones apriorísticas, cabe determinar más ampliamente el carácter actual de la Teoría de la Educación, teniendo en cuenta su procedencia anglófila, para así saber cual es su contenido y también su propia razón de ser.

\section{HOY}

La Teoría de la Educación, tanto en el Reino Unido como en los Estados Unidos, se ubica en dos grandes campos educativos: el escolar y el no escolar. El estudio del primero de ellos se reconoce por Teoría del Curriculum (Theory of Curriculum) y el del segundo por Educación no formal (No Formal Education). Debemos no obstante acotar la anterior afirmación. La Teoría de la Educación no contempla todo el conocimiento posible sobre la cuestión curricular, ya que algunos aspectos no forman parte de su objeto (valores, filosofía, axiología, fines, aspectos antropológicos... etc. del curriculum), y por otra parte, apenas incide en lo que se viene en denominar Educación Informal, al no tener ésta un carácter intervencionista definido. Con ello estamos aproximándonos al carácter fundamental de la actual Teoría de la Educación y es su sentido pragmático, utilitarista, continuador en definitiva de la tradición que se iniciara ahora hace un siglo a través de la denominada Escuela de Chicago y su teoría dramatística acerca de la realidad (W. James, Peirce, J. Dewey... etc.). Téngase en cuenta que en inglés la palabra «education» se refiere exclusivamente a la realidad, a la práctica, a la acción o si se quiere, al fenómeno tangible y real de la educación; de ahí entonces que si se teoriza sobre la educación es para servir a la práctica, al ejercicio profesional del educar. En el mundo anglosajón, Teoría de la Educación es sin duda Teoría para la práctica educativa (o para el ejercicio de la práctica educativa). No se concibe una teoría educativa que no pueda incidir positivamente sobre la práctica; de hecho tal situación para una mentalidad anglosajona sería absurda. Se teoriza en educación para mejorar la propia educación.

En consecuencia, la Teoría de la Educación se centrará:

* en su ubicación escolar, en los conocimientos curriculares que más tengan que ver y que más próximos están a la práctica educativa de las escuelas

* en su vertiente no escolar, en los aspectos de la educación no formal de intervención más posibilista y necesaria (campo de la educación permanente, laboral de adultos, educación urbana... etc.). 
Por último, cabría definir el sentido que en el mundo anglófilo de la Teoría de la Educación posee y tiene el propio término «teoría». Brevemente puede decirse que Teoría se adscribe al concepto de cuerpo sistemático y coherente de conocimientos con capacidad de propiciar en su aplicación efectos esperados; o sea, que más o menos, si bien sin el rigurosismo epistemológico de la Teoría de la Ciencia, «teoría» se puede adscribir a «ciencia», al menos en el sentido con que se reconoce a las teorías de las actuales Ciencias Humanas.

En consecuencia, y como resumen, la Teoría de la Educación anglófila, originada tras la desmembración de la unitaria Pedagogía germánica, se conforma como un cuerpo específico de conocimientos, integrado en la amplia constelación de saberes acerca de la educación (Ciencias de la Educación), que el desarrollo científico-investigador del mundo anglosajón propició, fundamentalmente, tras la segunda gran guerra, y que tiene como características específicas las dos siguientes:

* Una concepción de la teoría próxima a los desarrollos logrados en el seño de las denominadas Ciencias Humanas.

* Una concepción de la teoría en cuanto que saber o conocimiento aplicable (a la realidad escolar o no) cuyo objetivo es la mejora (innovación, cambio...) de la práctica educativa.

\section{TEORÍA DE LA EDUCACIÓN. SENTIDO Y OBJETO}

Definir el sentido y objeto de la Teoría de la Educación presupone un ejercicio que acaso pueda parecer dicotómico pero que en todo caso tiene vocación unificadora. Me refiero al hecho de que si hablamos de Teoría de la Educación (y no de Pedagogía) debemos aglutinarnos a la mentalidad y tradición anglosajona que ha sido, por otra parte, quien ha posibilitado la existencia de la propia Teoría de la Educación así como su presencia entre nosotros, y al mismo tiempo, debemos adaptar esta presencia a nuestra realidad científico-comunitaria en el campo de los estudios educativos o pedagógicos.

Un análisis sucinto de nuestra realidad en el campo de la teoría educativa (monografías o libros publicados bajo tal denominación, contenidos de la revista Teoría de la Educación, temáticas generales, ponencias y comunicaciones de los congresos nacionales de Teoría de la Educación, así como los trabajos elaborados en los diez seminarios habidos de Teoría de la Educación), supone de principio acatar la siguiente afirmación: No todo el conocimiento teórico de la educación entra a formar parte en sentido estricto de la materia "Teoría de la Educación". Se dan, que duda cabe, conocimientos teóricoeducativos, tales como los de corte histórico o filosófico que no forman parte de la Teoría de la Educación; en este sentido la discriminación creo que no ofrece duda alguna: siempre que se trate de un conocimiento que no posea aplicabilidad directa o inmediata a/en situaciones de práctica educativa no se conformará nunca como conocimiento propio de la Teoría de la Educación.

En consecuencia, el área concreta del dominio propio de la Teoría de la Educación estará formada por todas aquellas aportaciones orientadas a conocer la realidad educativa a fin de intervenir (modificar, innovar... etc) sobre la realidad educativa, (conocer para mejorar la práctica educativa). 
Ello quiere decir que el espacio formal de la Teoría de la Educación viene dado por todo el conocimiento que se requiere para mejorar la práctica educativa, por lo que acepta e integra información y conocimiento originario de las Ciencias de la Educación y en general de cualquier otro desarrollo disciplinario que en un momento dado pueda significarse como pertinente para la mejora de la práctica educativa, por lo que una vez más nos encontramos con la concepción ya afirmada de que la Teoría de la Educación sólo tiene sentido si es teoría para la práctical.

Consecuentemente, para el logro de este conocimiento podrá aceptar verdades ya asentadas en otras disciplinas, preferentemente las denominadas Ciencias de la Educación, o intentar investigarlas en función de sus necesidades y objetivo práctico, por lo que aceptará cualquier metodología adecuada a sus fines o intereses, fundamentalmente la descriptiva, la experimental, la analógica (simulación) y la comparativista.

Así pues, al ser la Teoría de la Educación una sistemática de conocimientos que procurará la intervención para mejorar, innovar, modificar...etc. la práctica educativa, deberá propiciar normas de acción, lo que supondrá siempre ciertos niveles de concepción tecnológica, entendida en el sentido de Bunge, o sea, como aplicación racional, ya que

1. Es evidente que en nuestra exposición se van solapando la Teoría de la Educación tal como la planteo y la Didáctica, tal como se reconoce en España. Y es lógico, ya que ambas denominaciones al obedecer a épocas, mentalidades, tradiciones y contextos culturales diferentes no tendrían que convivir. Intentaré una brev explicación.

Hasta bien entrado los años setenta del presente siglo, las cátedras universitarias españolas de nuestra especialidad obedecían a un planteamiento coherente y monolítico, integrado en la tradición alemana; así, además de la Historia de la Pedagogía, se contaba con cátedra de Pedagogía General (en Barcelona) o Racional (en Madrid), que se encargaba del conocimiento teórico-reflexivo de la educación, tal como hemos visto en las páginas precedentes; en consecuencia, a partir de aquí se propició una cátedra (en Madrid) de Pedagogía Experimental (la «otra» metodología de acceso al conocimiento educativo) y de Didáctica General y Especial, encargadas de estudiar la aplicabilidad del conocimiento pedagógico, bien especulativo (en Madrid), bien experimental (en Barcelona), a la realidad educativa, que en aquellos años era eminentemente escolar. Tres cátedras entonces que de alguna manera abarcaban los diferentes tipos de conocimiento pedagógico (teórico y aplicado: Pedagogía y Didáctica) y las diferentes metodologías (racional o especulativa y experimental: Pedagogía General y Pedagogía Experimental).

El problema actual se ha propiciado con la L.R.U. y la creación de las áreas de conocimiento que acogen en su denominación y en su concepción la tradición germánica de la Didáctica, y la anglófila de la Teoría de la Educación, lo que conlleva a la confusión de campos y de contenidos. Es evidente que desde una posición anglófila la Teoría de la Educación engloba a la Didáctica, prueba de ello es que esta materia o rama del saber no existe en la Universidad norteamericana, o del R.U. y su área de influencia (Australia, Nueva Zelanda..., etc.), por lo que, desde la Teoría de la Educación no se deben propiciar discursos diferenciadores respecto de la Didáctica puesto que no hay nada que diferenciar.

Otros temas que en España poseen tradición «didáctica» (aprendizaje, motivación, transferencia... etc.) así como otros fenómenos procesuales concurrentes en el binomio enseñanza-aprendizaje, son hoy en dia objeto de la Psicología de la Educación. También cabe advertir que debido a la gran incidencia e importancia que en la actualidad ha tomado el tema del curriculum, muchos departamentos de universidades de tradición anglófila incluyen en sus rótulos el sentido «curricular», en vez del de Teoría de la Educación, lo que no supone integrarse en un ámbito didáctico (que no existe). En fin lo que ha ocurrido en España, es que en Teoría de la Educación se asumió la denominación inglesa y se continuó con la tradición germánica, y en Didáctica, por el contrario, se siguió con la denominación germánica y se asumieron los contenidos anglófilos. Una reordenación de la situación, atendiendo a la tradición anglófila supone plantear dos grandes áreas de conocimiento por lo que se refiere a los estudios educativos: La Teoría de la Educación y la Psicología de la Educación, independientemente de la existencia de otras aproximaciones (Filosofía de la Educación, Historia de la Educación... etc.). 
incluso la no intervención (no directividad, autogestión, laisser faire, hegemonía del grupo... etc.) presupone una forma de intervenir, estudiada igualmente por las ciencias humanas, por lo que se pueden esperar resultados asimismo previstos.

En definitiva, lo visto hasta ahora podría resumirse esquemáticamente de la siguiente forma:

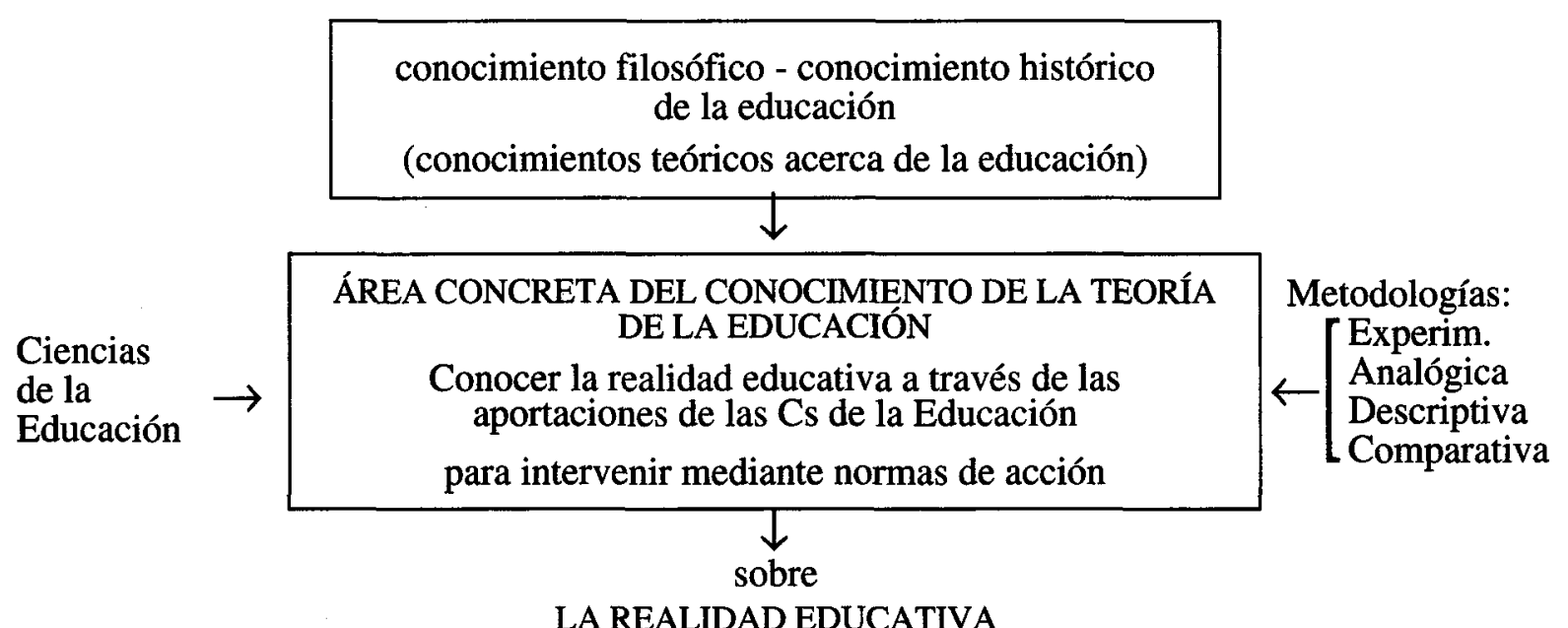

La concreción de las aplicaciones del conocimiento propio de la Teoría de la Educación o áreas en donde ubicar su acción -los espacios de aplicación, en definitiva- serían, para seguir con la tradición anglófila de nuestra materia, los denominados por $\mathrm{Ph}$. Coombs, como Educación Formal, Educación no Formal y Educación Informal. Así pues, la aplicación del conocimiento teórico educativo propio de la Teoría de la Educación al mundo de la educación escolar oficializada jurídicamente por las legislaciones estatales (Educación Formal), desarrollaría la Teoría de la Educación hacia el núcleo de conocimientos curriculares (Teoría del Curriculum), centrándose, fundamental y específicamente, en aquellos elementos de la Teoría Curricular más determinantes a la hora de posibilitar acción educativo-formativa, por lo que se incluye el campo instructivo ya que desde Herbart sabemos que el único soporte de la formación o educación se encuentra en los procesos instructivos, léase hoy en dia, a la luz de la Psicología de la Educación, el binomio enseñanza-aprendizaje.

No obstante, la Teoría del Curriculum, o los conocimientos acerca del curriculum, deben verse como un campo interdisciplinar, o de convergencia, de múltiples materias y desarrollos disciplinares y en absoluto como patrimonio de una sola de ellas, tal como se ha pretendido entre nosotros. De bote pronto podemos concebir la cuestión curricular como un espacio cognoscitivo en el que al menos pueden incidir y aplicarse a él los historiadores de la educación (la Historia de la Educación), los filósofos o antropólogos de la educación (la Filosofía y la Antropología de la Educación), los teóricos de la educación (la Teoría de la Educación), los teóricos del aprendizaje (la Psicología de la Educación), los planificadores, organizadores y políticos de la educación (la Planificación, la Organización y la Política de la Educación)... etc. O sea, y de acuerdo con las áreas de conocimiento en que está organizada la Universidad española, la mayor parte de las perspectivas posibles de estudio del curriculum, entran de lleno dentro de la denominada Teoría e Historia de la Educación. Y con ello no quiero atribuir a un argumento admi- 
nistrativo categoría cientítica, sino simplemente advertir de una realidad propiciada por la inclusión en nuestro contexto de la terminología anglófila -Teoría de la Educaciónque deja vacía de contenido (o casi) a la Didáctica (de tradición germánica) y que en cambio sí tenía sentido, antes, cuando convivía con la Pedagogía, al ser ésta de cariz más reflexivo y racional, fundamentalmente en su metodología.

Como habíamos advertido, el segundo campo de aplicación de la Teoría de la Educación se encuentra en la denominada Educación no Formal, o sea, en todos aquellos procesos educativos-formativos no desarrollados por la realidad educativa a la que hemos denominado escuela, propia de la Educación Formal. Pues bien, en el campo de la Educación no Formal, podemos distinguir dos grandes áreas de aplicación en función de la finalidad educativa con la que dotamos nuestros procesos, a saber la social y la ambiental. Así, de la aplicación de los conocimientos propios de la Teoría de la Educación a ambientes no formales de educación, con intención de logro de mejorar la formación, condición... etc. social de los individuos objeto de nuestra aplicación, surgirá lo que hoy en día viene llamándose Pedagogía Social (si no cambiásemos la terminología anglosajona tendríamos que denominarla Teoría de la Educación Social o simplemente tal como se hace Educación Social), y de un proceso paralelo aplicado empero a la mejora del medio ambiente nace lo que se denomina Educación Ambiental.

Por último, la aplicación de la Teoría de la Educación al ámbito de la Educación Informal, nos posibilita el tercer gran campo de desarrollo que ya habíamos anunciado, si bien en este caso la vocación intervencionista y pragmatista de la Teoría de la Educación queda de hecho cercenada pues como se sabe lo informal en educación es aquella situación que sabemos educativa pero sobre la cual no podemos intervenir bajo reglas o normas prescritas. No obstante ello no implica que se pueda estudiar y analizar, por lo que la reconocemos como objeto de la Teoría de la Educación.

Esquemáticamente el campo de aplicación de la Teoría de la Educación sería el que, como prolongación del primer esquema, desarrollamos a continuación:

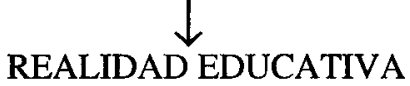

ESPACIOS DE APLICACIÓN DE LA TEORÍA DE LA EDUCACIÓN

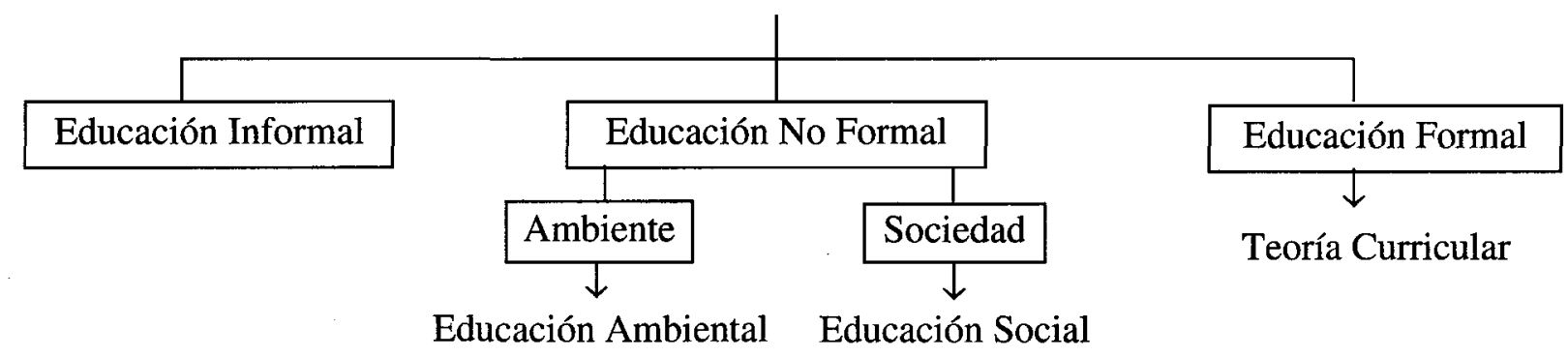

Por último, decir que la comprensión de toda la realidad educativa (teórica y práctica), y en consecuencia, de todo lo mencionado desde posiciones epistemológicas daría lugar a la Epistemología de la Teoría de la Educación, y en consecuencia a un discurso propio del mundo tres de Popper (en cambio la Teoría de la Educación, al incidir sobre realidades educativas se mueve en el segundo universo popperiano). Decir que en nuestro particular ámbito se han desarrollado visiones epistemológicas, fundamentalmente, desde la teoría sistémico cibernética, la filosofía de la tecnología, la filosofía analítica y 
la filosofía crítica. Asimismo la fenomenología y en consecuencia la obra de Husserl, así como algún que otro desarrollo propio de la última teoría de la ciencia, han tenido también algún eco entre nosotros.

\section{A MODO DE CONCLUSIÓN}

La Teoría de la Educación en España se integra entre nosotros en la segunda mitad de la década de los setenta y como consecuencia de la abertura que la educación española realiza tras la aprobación de la ley general de educación de 1970 y el consecuente desarrollo de las denominadas Ciencias de la Educación. Este hecho producirá una situación de choque que ha ido bloqueando en parte su desarrollo entre nosotros. La gran tradición española en estudios de «Pedagogía» y la mentalidad germánica de los mismos, más especulativa, antropológica y reflexiva, son solapados sin solución de continuidad por una rutilante Teoría de la Educación, que por lo general ha conllevado a unos y a casi todos los otros a continuar «haciendo» Pedagogía o epistemología, o cualquier otra cosa bajo la nueva denominación. Como conclusión de todo lo expuesto diré que considero falaz e inutil las adscripciones del tipo «Pedagogía igual a Teoría de la Educación», puesto que la cuestión no es tanto de contenidos o espacios conceptuales cuanto de mentalidad a la hora de enfocar estos contenidos. Profesar la Teoría de la Educación supone, debe suponer, como acto de prima coherencia, cambiar de mentalidad a la hora de enfocar las cuestiones educativas; la Teoría de la Educación, por su origen anglosajón rezuma toda la tradición pragmatista y utilitarista propia de estos paisajes, en contra entonces de la corriente más racional, fundamental, reflexiva y filosófica de la Pedagogía alemana (cuya tradición se encontraría hoy más en el campo de la Filosofía de la Educación). El teórico de la educación, en tanto que profesa como campo de estudio e investigación la Teoría de la Educación, debe encarar la educación desde la mentalidad pragmática, anglófila en la que se asienta su materia; de ahí que el nudo gordiano de la «Teoría de la Educación» sea conocer para hacer. En efecto, en Teoría de la Educación debe darse un conocimiento acerca de la realidad educativa, propiciado por lo general (pero no exclusivamente) por las denominadas Ciencias de la Educación, a través de diversas y pertinentes metodologías (descriptiva, experimental comparativista y analógica, fundamentalmente), a fin de que este conocimiento de la realidad nos posibilite formas de intervención adecuadas y eficaces para modificar o mejorar la situación de la educación. Es, y vuelvo a reiterarlo, el saber para saber hacer, el conocer para hacer, que encuentra en la Educación Formal, en la Educación no Formal y en la Educación Informal sus campos de aplicación, y como desarrollos consecuentes de esta aplicación, la Teoría del Curriculum, la Educación Social y la Educación Ambiental, fundamentalmente. En consecuencia, la estructura conceptual de la Teoría de la Educación vendría dada por los siguientes niveles de conocimiento:

* Nivel previo de carácter metateórico: Concepción de la realidad (educación), del conocimiento de esta realidad (epistemología), y de las formas para acceder a esta realidad (procesos heurístico-investigacionales y metodologías pertinentes).

* Nivel teórico-científico: Aportaciones propias, de las Ciencias de la Educación y de otras disciplinas a fin de conocer la realidad educativa sobre la que se debe intervenir. 
* Nivel tecnológico de aplicación:

Educación Formal: Teoría del Curriculum y cualquier cuestión que afecte a la práctica educativa escolar, siempre que sus planteamientos se conciban desde una perspectiva científica y por tanto teórica.

Educación no Formal: Educación Social, Educación Ambiental.

Educación Informal.

De ahí que consideremos a la Teoría de la Educación como una materia de carácter teórico-tecnológico (Teoría Tecnológica), independientemente del sentido paradigmático al cual nos adscribamos. No se debe confundir aquí un proceso «tecnológico» 0 «crítico» o «hermenéutico», con el carácter que la Teoría de la Educación tiene de conocer (Teoría) para hacer (Tecnología), ya que la tecnología propia del hacer (hacer racional, fundamentado en formas de conocimiento racional) son propias de los procesos críticos (sistema de alfabetización de P. Freire), tecnológicos en sentido estricto (enseñanza asistida por ordenador) o de corte hermenéutico (estrategias etnográficas).

Tarragona, febrero, 1992 